\title{
LIFELONG SELF-DIRECTED LEARNING IN THE DIGITAL AGE: AN ORIENTATION OF CURRENT SOFTWARE TOOLS SUPPORTING EXPERTS IN MAINTAINING AND UPDATING THEIR KNOWLEDGE
}

\author{
Jodie Freeman $^{1}$, Andreas Raabe ${ }^{1}$, Felix Schmitz ${ }^{2}$ and Sissel Guttormsen ${ }^{2}$ \\ ${ }^{1}$ University Clinic for Neurosurgery, Neuro Centre, University hospital of Bern, Switzerland \\ ${ }^{2}$ Institute of Medical Education, Medical Faculty, University of Bern, Switzerland
}

\begin{abstract}
To guarantee high-quality services, health professionals are required to successfully maintain their extensive knowledge base. $\mathrm{M}$, health professionals are forced to consistently stay up-to-date in their field in which new knowledge is evolving continuously. Hence, there is a strong need for effective support during their lifelong, self-directed learning processes as a means of maintaining and updating medical-related expert knowledge. From the literature, self-organisation, -management, -assessment, and collaboration activities have been defined to be of high relevance for these learning processes.

The increasing speed of technological development has led to the development of a plethora of digital tools with varying features aimed at supporting professionals in their self-directed learning. In light of the increased number of tools available, we aim to give orientation on some of the most popular tools available to support health and other professionals in their self-directed learning. Results show that three main categories of digital tools related to self-directed learning can be identified: reference-management systems, learn-and-test tools, and collaborating tools. Our results further show that within the current landscape, there is no one tool that covers all tasks related to self-directed learning. We conclude that one single digital tool, combining all the features supporting self-directed learning in one simple easy to use manner may better support health and other professionals in maintaining and updating their expert knowledge. Future development should focus in particular on suitable combinations of features supporting self-directed learning, as implemented within one tool.
\end{abstract}

\section{KEYWORDS}

Self-Directed Learning, Health Professionals, Software Tools

\section{INTRODUCTION}

Health professionals are required to stay up to date in their field in which new knowledge is evolving continuously (Kompen, 2019). In addition to updating new areas of expertise, health professionals also have to maintain their existing knowledge base enabling them to be lifelong learners (Teunissen and Dornan, 2008). A systematic review (Murad et al., 2010) identified self-directed learning (SDL) as the most appropriate methodology for maintaining and updating levels of expertise and promote lifelong learning; hence SDL has been advocated as an effective and efficient activity for health professionals (Kompen, 2019). Aspects of SDL include self-analysis, self-assessment, self-evaluation and collaboration (Costa and Kallick, 2004). Learners who self-assess themselves enhance continuous lifelong SDL in which technology is reducing the effort needed to find, organise, manage and interact with information (Costa and Kallick, 2004). The emerging problems and challenges for experts practicing SDL are manifold. For example, learners must quickly identify relevant digital information from insurmountable range of sources coupled with the need to store, manage and retrieve these documents quickly and efficiently, in which the traditional means of managing information (e.g. individually storing data in electronic filing systems) are ill-suited to the realities of the digital age (Bryan, 2015). Current restrictions in working hours and changes in the organisation of health care make it ever more necessary for health professionals to utilise their time productively and learn more efficiently (Teunissen and Dornan, 2008). 
The digital age is profoundly changing the ways in which we learn with the use of technologies (Kompen, 2019), in particular how we search, select, use and interact with information for our professional development. Printed textbooks and journals are increasingly being replaced by PDF versions and additional digital sources, such as videos, web pages, blogs, etc. Developments in digital technology, and the speed at which they emerge, drive innovation and new applications that touch our lives in different and often profound ways (Khosrow-Pour, 2018). Given the increased use of fast changing digital technologies in the workplace, new skills needs have emerged (Khosrow-Pour, 2018). The use of these technologies has contributed to transforming learning and skills development into a lifelong process. From this perspective, learners have to continually improve and evolve with technology to benefit from the support in their learning. Manganello et al., (2013) found that many software tools require training to fully exploit the learning power of these technologies, highlighting that it may be more beneficial to develop a single, easy to use interface, which provides the SDL functionalities needed to support learning without having to master complex technological skills.

The increasing speed of technological development has led to the development and advancement of a plethora of digital tools that claim to support learning and combat the challenges the increasing rate of information has brought about. There is a lot more recognition in SDL for learners to utilize technology and the current research shows a growth in digital software aimed at the various learning needs of professionals (Kompen et al., 2019). Research highlights that digital tools can support SDL in many ways, helping learners skillfully "access the expanding house of knowledge in the Web 2.0 and beyond environment" (Bryan, 2015. $\mathrm{Pg}$. 44). Hence, digital tools can also increase the speed of accessing information, increase confidence in the learner and responsibility for what they learn. In addition, with digital tools learners have the potential to collaborate, share content and establish new online communities, which support SDL both, in formal and informal settings (Bryan, 2015). However at present, there is no one tool that assists learners in reordering, classifying, interacting with proper knowledge resources and sharing them with learners with similar needs thus more than one digital tool is needed to support their SDL needs (Manganello et al, 2013). Therefore, the aim of this paper is to give orientation on some of the most popular current digital tools used by professionals to support their SDL. We also seek to show which precise features of those tools actually correspond with SDL-related principles. Hence, these research questions (RQ) are formulated:

RQ1: Which digital tools are used most frequently by health professionals in order to support their SDL?

RQ2: Which of those tools are made up of features linked with SDL-related principles as mentioned in the existing literature?

\section{METHODS}

To give an overview of current digital tools' SDL-related features, it firstly had to be defined, which tools should be analysed according to our questions. Based on existing opinions of researchers $(n=5$ experts at our university clinic) a selection of tools used by professionals were identified. We identified 9 Tools that could be clustered as management systems, learn-and-test tools, and collaborating tools (e.g. for writing a paper together). To further validate our first selection of tools, a comparison was made with the learning tools available amongst students and post-doctorates as identified by the Head of Library and research services at the University of Bern. The selection of tools as set out in this paper were confirmed as current tools that support learning within research institutions. The most frequently named tools were:

- Endnote (https://endnote.com/) as representative for reference-management systems

- Authorea (https://www.authorea.com) as representative for collaboration tools

- Brainscape (https://brainscape.com) as representative for learn-and-test tools

In a second step, we used the data bank AlternateTo (https://alternativeto.net/); with this data bank one can query software tools that have comparable features to a respective competitor. Importantly, the query results are ranked based on an online-community, which means that the higher a tool is listed the more favourable it is rated by the community. Accordingly, we searched for alternatives for the three mentioned tools - and chose the two first, and thus most popular, digital tools per category in the list of the query results.

In a third step, the features offered by the resulting nine digital tools (see Table 1) were independently analysed by two experts along the following SDL-related tasks: (i) finding, storing, retrieving information, 
(ii) self-organisation, (iii) self-management, (iv) self-assessment, and (v) sharing/collaborating. According to Costa and Kallick (2004), these tasks refer to as the most central components of SDL.

In the fourth step, directly after the ratings were completed, the two raters were tasked with reaching a consensus in the case of differences in their ratings. They discussed the few cases in which features were similar to reformulate them into one single feature and excluded features when agreed that they are irrelevant to the domains of SDL. Based on consensus between the raters, final univocal allocations were determined.

In the fifth and final step, we compared the digital tools by opposing their SDL-based features in a matrix.

\section{RESULTS}

The findings highlight that there is no single tool that offers all SDL-related features linked with supporting self-directed, lifelong learning. Instead the tools offer a set of features that support one or two certain component(s) of the learning process. For details, see Table 1.

Table 1. Prominent software tools and their SDL-related features

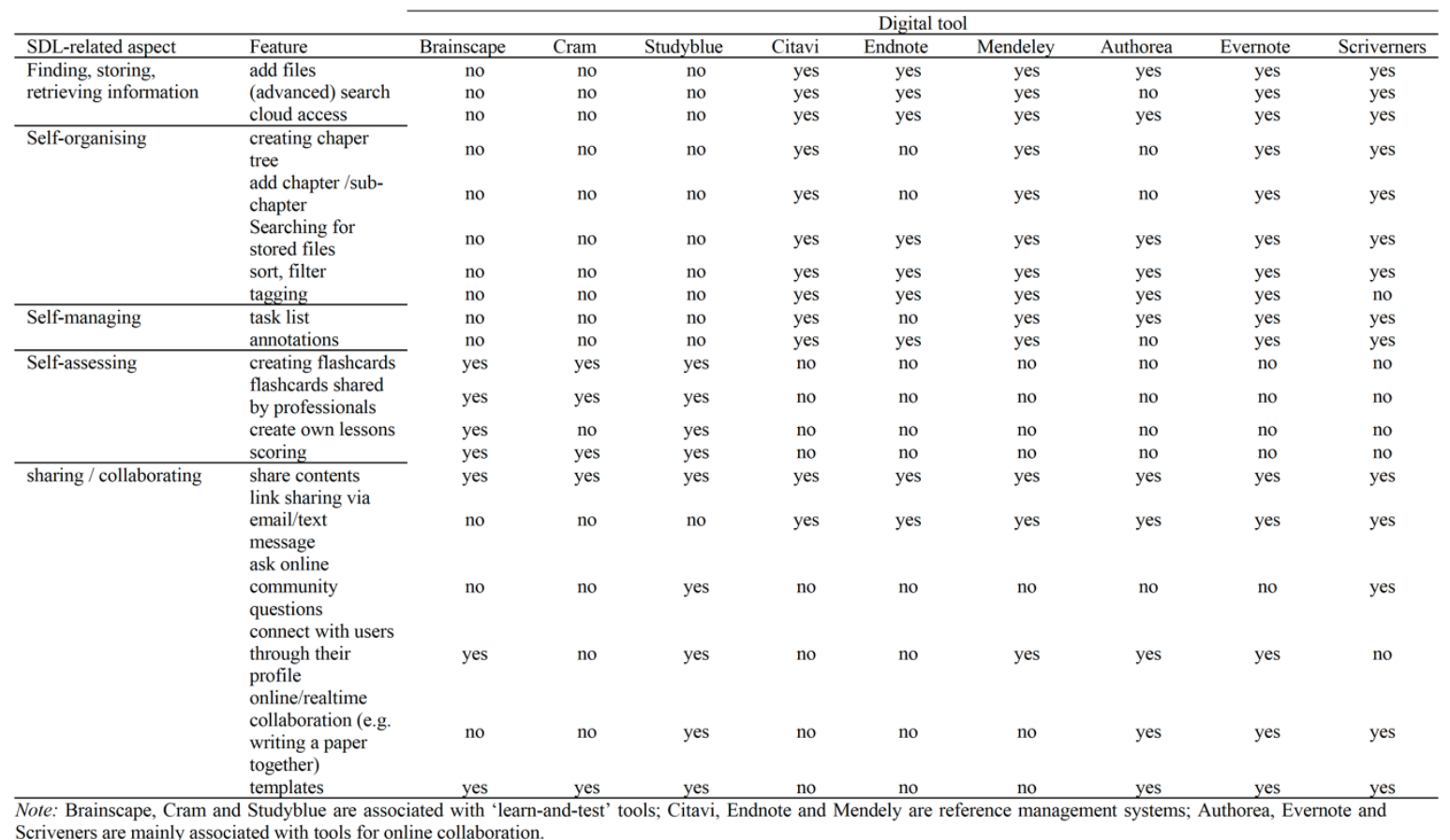

\section{DISCUSSION}

Within this study, we provided an overview of the most popular digital tools for health professionals, which are used for SDL-related purposes. Our two research questions are clarified: Firstly, the questioned health professionals most frequently use reference-management systems for their daily work. Nonetheless, they also use collaboration and 'learn-and-test' tools. Secondly, the tools that we evaluated only offer a set of features that support one or two component(s) of the SDL process. These components specifically include knowledge management, self-assessment and collaboration, which seem to address major needs of health professionals.

Our findings show that there are a range of digital tools available to support professionals in their learning, with different tools offering a variety of features. However, based on our main finding, the tools we analysed do not offer a combination of features covering all the aspects of SDL at once. The need to support various functions within one tool is supported by previous literature denoting the importance of combining a range of varying features to create a successfully functional personal learning environment (Attwell, 2007) that supports SDL in a holistic sound manner (Kompen, 2019). Each digital tool analysed in this study supports one or two component(s) of SDL and therefore a varying choice of tools and their features (which in 
turn support different components of SDL) would need to be combined in response to individual learning needs, aims and goals (Attwell, 2007). We assume that health professionals are not supported optimally, when they have to use a set of various tools in order to be fully supported in their SDL, further research should explore this assumption.

Further, it should be investigated, which functions of the SDL activities ideally can be implemented in one single tool, without resulting in an overload of functions and low usability. Previous findings show that when technology aimed at supporting the user get too complex, the acceptance of the learner suffers (Khosrow-Pour, 2018), suggesting that a tool, which combines the various features for how we store, manage, assess knowledge, and collaborate with others may be too complex to handle resulting in reduced efficiency. In addition, if digital tools are over complicated with too many features, research shows that learners become frustrated and spend more time learning to use the tools than actually learning from relevant materials (Khosrow-Pour, 2018).

Taken together, it might be worthwhile to design a digital tool that, on the one hand, offers a combination of all features of SDL (cf. Costa and Kallick, 2004). On the other hand, the concept of such a tool must also focus on the tool's usability in order to reduce the time spent learning the software as well as to make it easy and satisfactory to use (Teunissen and Dornan, 2008). Consequently, future research should focus on how to design digital tools that include all central components of SDL by also providing a strong alignment with the actual needs and ICT competencies of health professionals (Teunissen and Dornan, 2008).

\subsection{Limitations}

This study has some limitations. Foremost, we were not able to systematically identify all digital tools that, at present, address SDL-related features. Findings are based on a selection of corresponding tools. Still, the selection made in this study is likely to be representative, as it is based on the typical target population and their habits, as well as on a much broader online-community. We conclude that our results are generalisable.

Another potential limitation is that the scope of the selected tools also represents an inherent selection of features. For instance, a reference-management system is normally not built for self-assessment reasons. Nonetheless, this issue does not derogate our main finding that there is no one prominent tool available at present that covers all central components of SDL; users only use digital tools available on the current market.

\section{CONCLUSION}

This study offers an orientation of prominent digital tools used by health professionals for SDL-related reasons. Most importantly, we could not identify a single digital tool that covers all components of SDL as discussed in the literature. Hence, future research should focus on how to design a tool that offers more features linked with SDL-related components in one solution - by bridging the gap between usability, knowledge management and human learning principles, also addressing the domain-specific needs of health professionals.

\section{REFERENCES}

Attwell G., 2007. Personal learning environments: the future of eLearning? Elearning Papers, Vol. 2, No. 1, pp 1-8.

Bryan V.C. 2015. Self-directed learning and technology. The Education Digest, Vol. 80, No. 6, pp 42-44.

Costa, A. L. and Kallick, B., 2004. Assessment Strategies for Self-Directed Learning. Sage Publications, California.

Khosrow-Pour M., 2018. Handbook of Research on Challenges and Opportunities in Launching a Technology-Driven International University. Advances in Higher Education and Professional Development. IGI Global, USA.

Kompen T. et al, 2019. Personal learning Environments based on Web 2.0 services in higher education. Telematics and Informatics, Vol. 38, pp 194-206.

Manganello, F et al, 2013. PKS: An ontology-based learning construct for lifelong learners. Journal of Educational Technology \& Society, Vol 16, pp 104-117

Murad, M.H et al, 2010. The effectiveness of self-directed learning in health professions education: a systematic review. Medical Education, Vol 44, No 11, pp1057-1068

Teunissen, P. W. and Dornan, T., 2008. Lifelong learning at work. British Med. Journal, Vol. 336, No. 7645, pp 667-669. 